\title{
«Wir wollen uns einordnen - nicht unterordnen»
}

\section{Daniel Lüthi}

Freier Journalist und Fotograf, Medientrainer, Bern

Schönberg: Der Name stimmt nicht ganz. Besonders schön ist hier nämlich nicht der Berg, (ein Hügel eher), sondern die Aussicht. «Die Perspektive», wie Marcel Glässner immer wieder betont. Das 180-Grad-Panorama ist atemberaubend. Der Weitblick reicht von Merligen über das Dreigestirn Eiger, Mönch und Jungfrau, die Simmenfluh und die markante Niesenpyramide bis fast nach Thun. Im Vordergrund immer der See, der Stimmungsschwankungen widerspiegelt und oft auch nur beruhigend da liegt.

\section{Zwischenstation}

Die Klinik Schönberg besteht aus mehreren Häusern im Chalet-Stil. Sie feiert dieses Jahr ihr 25-jähriges Bestehen. «Dies ist eine Klinik mit einem gehobenen Hotelkomfort», sagt Marcel Glässner. "Ärzte, Physio und Pflege sind hier das Dreigestirn.» Im Restaurant werde der Patient wie ein Gast in einem Restaurant behandelt, und nicht wie der Bewohner eines Heims. Wie werden die temporären Bewohner hier denn genannt?

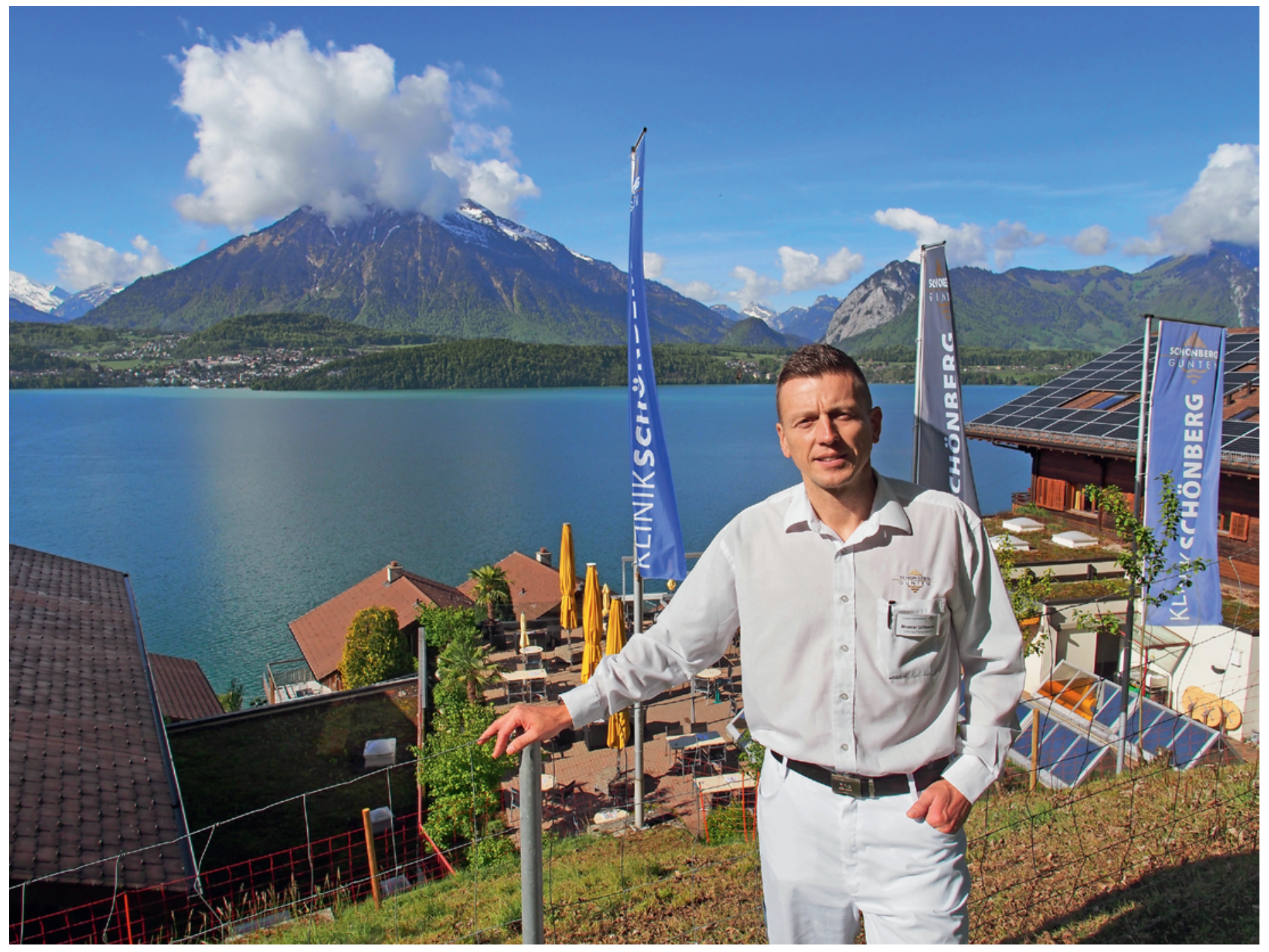


Sind sie "Patienten», "Klienten» oder "Gäste»? "Eine Diskussion, die andauert», antwortet Glässner. «Sicher nicht «patients`, Geduldige im ursprünglichen Sinn; geduldig muss vor allem ihr Umfeld sein.»

Eine Rehaklinik ist für die Patientinnen und Patienten eine Zwischenstation - zwischen einem Akutspital und dem heiss herbeigesehnten Zuhause. Oder einer Langzeitinstitution. «Nicht nur ein Arm oder Bein sind hier, sondern Patienten mit einem oft komplexen Umfeld. Wir begleiten sie in einem Prozess.» Als exemplarisches Beispiel nennt er den Klavierspieler mit dem gebrochenen kleinen Finger. «Für ihn ist diese Verletzung eine Tragödie - er hat deshalb auch ein spezielles Schmerzempfinden.»

\section{"Ärzte, Physio und Pflege sind \\ hier das Dreigestirn.»}

Vor allem älteren Menschen begegnet man hier, viele davon werden in Rollstühlen durch die Verbindungsgänge und das Areal gestossen. Einige haben bei einem Unfall Knochen gebrochen, andere haben ein neues Hüft- oder Kniegelenk erhalten. Wer schon so weit ist, profitiert von den physiotherapeutischen Einrichtungen und Dienstleistungen, andere sitzen auf dem Balkon, sehen zu, wie der Raddampfer «Blüemlisalp» vor dem gleichnamigen Bergmassiv wie ein Postkartensujet seine Spur durchs Wasser zieht - und warten aufs Mittag- oder Abendessen.

\section{Bezugspflege}

Dazwischen sind immer wieder Glässners Leute aktiv: Rund 80 Personen arbeiten unter seiner Leitung in der Pflege, dazu gehören die Stationsleitungen, der Sozialdienst, die Wundversorgung und die Apotheke. «Bei uns geht es im Schwerpunkt nicht um Medizinaltechnik», kommentiert Glässner, "wir wollen uns unserer Patientinnen und Patienten ganzheitlich annehmen. Es geht mir um eine Bezugspflege - darum, dass sich meine Mitarbeitenden verantwortlich fühlen.» In jedem Haus hängt eine Tafel mit den Fotos der Personen, die heute gerade zuständig sind. Vor drei Jahren, als er seine Stelle angetreten habe, seien die Zuständigkeiten nicht klar geregelt gewesen, erzählt Glässner. «Jetzt sehe ich langsam die Früchte meiner Arbeit.» Nämlich: «Dass jeder am Patientenbett seiner Ausbildung und seinen Skills entsprechend seine Leistung erbringt.» Und: "Dass wir alle dieselbe Sprache sprechen, wenn wir über unsere verschiedenen Pflegeinterventionen reden». Was genau heisst «Medikamente abgeben» oder "jemanden zum Restaurant begleiten»? Es sei wichtig, dass alle unter jedem Element

\section{Marcel Glässner}

Marcel Glässner wurde 1972 in Eisenach in der ehemaligen DDR geboren. 1989, kurz vor dem Fall der Mauer, übersiedelte er in die Bundesrepublik Deutschland, wo er das Abitur machte. Von 1993 bis 1996 liess er sich zum diplomierten Pflegefachmann ausbilden. 1998 übernahm er bei der Universitätsklinik Düsseldorf


die Co-Leitung einer kinder- und jugendpychiatrischen AkutAufnahmestation. Bis 2002 absolvierte er berufsbegleitend eine Leitungsausbildung. Ab 2007 leitete er die Alpine Kinderklinik Davos, die 2009 geschlossen wurde. 2009 bis 2013 war er Bereichsleiter Pflege im Berner Rehazentrum Heiligenschwendi, seit 2014 leitet er den Pflegedienst der Rehabilitationsklinik Schönberg in Gunten. Marcel Glässner ist verheiratet und Vater von zwei kleinen Söhnen. Er lebt mit seiner Familie in Heiligenschwendi.

dasselbe verstehen, sagt Glässner, «es geht um eine Standardisierung, und es geht um Effizienz. Im Vordergrund steht die Pflegequalität, aber natürlich können wir unsere Parameter auch für das Controlling oder die Abrechnung nutzen - denn hinter jeder Handlung steht eine Minutenzahl. Ab 200 Minuten pro Tag gilt jemand bei uns als aufwendig."

In der Wahrnehmung der Gesellschaft habe die Pflege in einer Reha-Klinik im Vergleich zum Akutspital lange als weniger anspruchsvoll gegolten. «Die Fallpauschalen haben dies - unter anderem - verändert. Der Kostendruck der Vor-Institutionen wächst, diese interpretieren die Realität bisweilen zum eigenen Vorteil, und oft kommt es zu verfrühten Entlassungen. Einige Male erlebten wir sogar eine Rückverlegung am ersten Tag.»

\section{Kompetenzgerangel}

«Ich bin Pfleger», sagt Marcel Glässner mit spürbarem Stolz. Auch wenn er nicht mehr so oft am Patientenbett steht. «Das ist ein eigenständiger Beruf, wir sind keinesfalls bloss die Handlanger der Ärzte. Wir wollen uns

\section{«Ab 200 Minuten pro Tag gilt jemand bei uns als aufwendig.»}

einordnen - nicht unterordnen.» Ab und zu erlebe er zwischen den verschiedenen Berufsgruppen leider immer noch ein Kompetenzgerangel, an Rapporten zum Beispiel, aber auch im Beisein von Patienten: «Die Visite als Halbgott-Dämmerung, bei der alle ehrfürchtig den Hut vor dem weissen Kittel zu ziehen haben - das ist noch nicht ganz vorbei.» Glässner befürwortet denn auch die Volksinitiative «Für eine starke Pflege»: «Ärzte sollen und können Verantwortung an uns Pflegende 
abgeben. Heute müssen sie viel für uns entscheiden, was nicht nötig ist.»

Schon früh in seiner Karriere wollte Glässner personalpolitische Verantwortung übernehmen. «Nur beratend tätig zu sein, war mir ein bisschen zu wenig», erklärt er, «ich habs schon auch gern etwas direktiv.» Und so ist er heute auch Chef, Organisator mit betriebswirtschaftlichem Interesse, und ansatzweise sogar Pflegewissenschaftler. «Unser Schwerpunkt ist die muskuloskelettale Rehabilitation mehrheitlich bei Multimorbiden", erklärt er. Was genau dies im Einzelfall bedeute, sei vor seiner Zeit hier noch unklar gewesen: «Jeder hat sich gemäss seinem Hintergrund etwas gedacht - und darauf basierend etwas gemacht.»

\section{"Ärzte sollen und können Verantwortung an uns Pflegende abgeben.»}

Glässner hat die Pflegeinterventionen durch sprachliche Anpassungen nahezu halbiert und das Ganze zu den in dieser Klinik definierten 16 häufig anzutreffenden Pflegediagnosen gebündelt. "Haut- und Gewebeschädigung" ist eine solche Diagnose, "Naht-/Hautkontrolle» oder "VAC-Verband» sind darauf basierend mögliche Interventionen. «Wichtig ist bei allem die wöchentliche Zielüberprüfung - denn was sich nicht überprüfen lässt, können wir uns auch gleich sparen. Die Leistungserfassung gehört zur Pflege wie der Hammer zum Schreiner. Wir müssen dokumentieren, was wir gemacht haben, sonst gilt es als unerledigt.»

Zurzeit wird das neue System - «Pflege nach Pflegediagnosen» - in das Krankenhaus-Informations-System KIS implementiert. «Ich verknüpfe jede Intervention mit einem Code, der allen zeigt, welche Kompetenz dafür erforderlich ist. So sieht die Stationsleitung sofort, wen sie in ein Patientenzimmer schicken muss. Und natürlich vereinfacht dies auch die Personalplanung.» Pro Haus (mit je rund 20-30 Patientinnen und Patienten) und Tag ist - nebst einer FaGe, einer Assistentin sowie eventuell einer Praktikantin - eine diplomierte Pflegende eingeplant. "Sie kommen an die Grenzen, haben Stress. Deshalb habe ich pro Haus eine Diplomierte mehr beantragt. Dies hat die Direktion genehmigt, und ich bin ihr dankbar dafür.»

\section{Marathonläufer}

Marcel Glässner ist ein Kämpfer über lange Distanzen ein Marathonläufer. Die klassische Strecke über gut 42 Kilometer lief er auch schon in 2 Stunden 45 Minuten, was ihn damals, vor 12 Jahren, zum 2. Deutschen Meister seiner Altersklasse machte. Der Sport habe ihm 2007 die Auswanderung in die Schweiz erleichtert, sagt er: «Das war mein Schlüssel zur anderen Kultur.» Zweimal absolvierte er hier einen Ultramarathon über 80 Kilometer und 2500 Höhenmeter. «Diese Extremerfahrungen möchte ich nicht missen", sagt Glässner. Immer mehr komme er vom leistungsorientierten Sport aber weg. Das habe er unter anderem seinen zwei Kindern - drei- und einjährig - zu verdanken: «Meine beiden Jungs holen mich immer wieder runter. Sie ermöglichen mir einen Perspektivenwechsel: Bei ihnen muss ich nicht immer zwei, drei Schritte voraus sein.

\section{«Meine beiden Jungs holen mich immer wieder runter.»}

Sie leben im Hier und Jetzt. Und helfen mir auch, meine Schwächen zu erkennen - meinen stark ausgeprägten Perfektionismus zum Beispiel.»

Aber auch sein Körper beginnt Marcel Glässner Grenzen zu setzen. So stehen neue Hüftgelenke zur Diskussion. Und damit eine neue Art von Perspektivenwechsel: «Wer weiss», scherzt der Pflegedienstleiter, «vielleicht werde ich in dieser Rehaklinik dereinst selber Patient sein».

Bildnachweis

Fotos: Daniel Lüthi 